\title{
Internet of Things: learning and practices. Application to Smart Home
}

\author{
Olivier Debauche \\ Computer Science Unit \\ University of Mons - FPMs \\ Mons, Belgium
}

\author{
Saïd Mahmoudi \\ Computer Science Unit \\ University of Mons - FPMs \\ Mons, Belgium
}

\author{
Mohammed Amin Belarbi \\ Computer Science Unit \\ University of Mons - FPMs \\ Mons, Belgium
}

\author{
Mohammed El Adoui \\ Computer Science Unit \\ University of Mons - FPMs \\ Mons, Belgium \\ Email: mohammed.eladoui@umons.ac.be
}

\author{
Sidi Ahmed Mahmoudi \\ Computer Science Unit \\ University of Mons - FPMs \\ Mons, Belgium \\ Email: sidi.mahmoudi@umons.ac.be
}

\begin{abstract}
Internet of Things is becoming widely present in our daily life. In fact, more and more devices able to interact together have been recently designed and launched in the market. Learning Internet of Things technologies is becoming unavoidable in education. In this paper, we propose a practical approach allowing to progressively learn, by practice the, essential concepts of Internet of Things applied to Smart Homes. From basic knowledge of $\mathrm{C}++$ language and the use of Arduino or its derived, students can develop skills and also smart applications in the field of Internet of Things.
\end{abstract}

Keywords-Internet of Things, IoT, Smart Home, practical work.

\section{INTRODUCTION}

Internet of Things is considered as the next information revolution, and we estimate the number of devices connected at horizon of 2020 at 50 billion [1]. The Internet of objects is fundamentally different from the Internet of machines. The Internet networks of objects are dynamic, distributed, low throughput and composed of large quantities of heterogeneous objects from a functional and technical point of view [2], [3].

The Internet of Things includes a wide range of various smart interconnected devices such as sensors, effectors, machines, autonomous devices, drones, intelligent cameras, etc [4]. Smart Objects are generally compact devices with communication capabilities, identified by name and address, with computing capabilities, powered by batteries or solar panels [2] [5]. Connected objects are dynamic and have self-adaptive capabilities. They can also be self-configurable and support interoperable communication protocols. These objects can be producers, data-consuming or endorse both roles. The data produced are mainly eventual and temporal3. Connected objects can take the roles of sensors, actuators, controllers, or activity monitors [6].
Nowadays, learning activities related to Internet of Things technologies present a great challenge for educational institutes. Furthermore, all Master specialization are not familiar with the required knowledges of Internet of things, electronics and related computing. Teaching Internet of Things for non-initiated public presents an important challenge. That is why; we propose in this paper to design a new protocol of practical works which lead progressively students from basic knowledge in Arduino programming and wireless communication to the interaction of connected objects together without human intervention.

The learning protocol dedicated to Internet of Things presented in this paper is developed for Architect Engineer Master Student at the University of Mons (Belgium). We propose to explore different lighting methods in order to gradually lead students to understand different concepts and methodologies used in the Internet of Things.

\section{RELATED WORK}

In literature, several learning devices have been proposed such as Gonzalez et al., which proposed in 2008 an architecture based on using a NOKIA 6131 NFC mobile phone and some RFID tags [7] in the aim to interact with physical space. Gmez et al. proposed in 2013 an architecture composed of integrates NFC, QRCODE technology identification, 3G and $4 \mathrm{G}$ communication protocols in order to interact with physical objects[8]. Fernandez et al. used in 2015 the devices of led RGB, Arduino and Raspberry PI for teaching Internet of Things to children from 10 to 12 years [9].

More recently, $\mathrm{He}$ et al. developed a learning device for undergraduate students composed of Raspberry Pi Arduino and a set of sensors supporting Zigbee [10]. 


\section{MATERIAL \& SOFTWARE}

Our learning hardware is composed of three material thematic packages:

- The first one is distributed individually or for groups of two students and contains Arduino material ;

- The second package contains Libelium material where the objective is to use wireless connections to link different devices ;

- The last thematic package is composed of a common connected material using Ethernet connections to actuate related devices.

The main devices that compose our material packages are:

1) Home Assistant Server: A Raspberry Pi 3 hosts the last release of Home Assistant9 which is a platform for monitoring equipment automation; written in python 3 under MIT license. We use Home Assistant which allows in one interface to observe, control and automate all connected devises in a house and contains natively a MQTT10 broker (HBMQTT ). MQTT is an extremely lightweight publish-subscribe machine-to-machine protocol. We have used Hassbian to install the last version of Home Assistant with complementary services on the Raspberry Pi 3.

2) Arduino devices: Each couple of students received an individual kit composed of an Arduino Uno, three channels Red Green Blue (RGB) Shield Velleman VMA01 (Fig. 1), monochromatic led, tri color led, set of resistors, breadboard and set of cable. The shield Velleman allows to control low voltage individual led or ribbons of led and compose different mix of primary colors to obtain choice color. A dimmer $220 \mathrm{~V}$ made by us (Fig. 2); connectable on Arduino allows to regulate the intensity level of $60 \mathrm{~W}$ incandescent bulb.

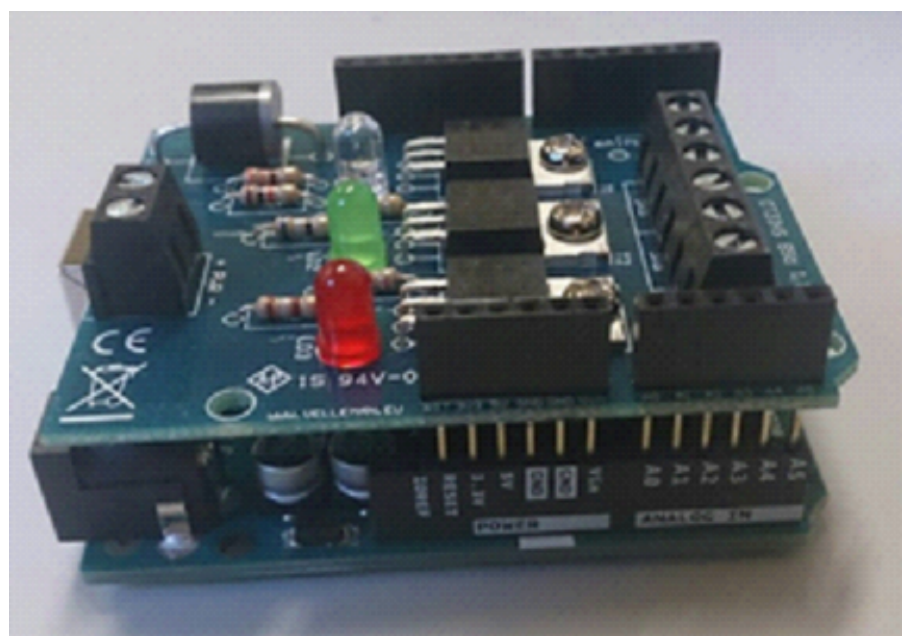

Fig. 1. Shield Velleman VMA01 mounted on Arduino Uno

3) Wireless material: We used three Libelium Evaluator Kit containing several Waspmote and additional shields to add specific capabilities to extend application domains

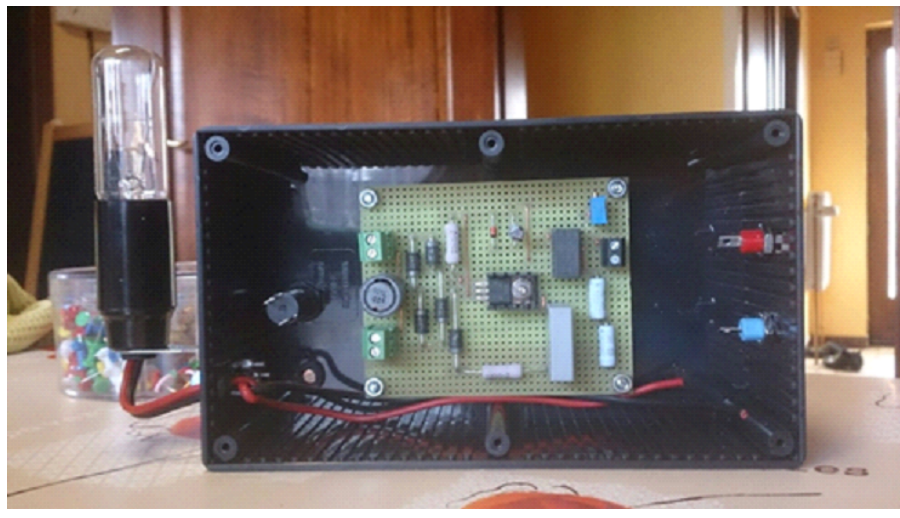

Fig. 2. Dimmer 220V

of individual Waspmote. The Waspmote is an Arduino modified for Internet of Things application by Libelium. Each evaluator kit contains also a supplementary Zigbee module allowing to Waspmote to communicate together

4) Actuation material: The actuation materiel that we used contains on one hand a Controllino Mega based on an Arduino Mega (microcontroller ATmega2560). The Controllino is especially connected on network with Ethernet interface and allows to activate a set of 16 relays which can manipulate $230 \mathrm{~V} / 6 \mathrm{~A}$. Moreover, it provides: 1 Real Time Clock (RTC), 1 Serial Peripheral Interface (SPI); 1 Inter-Integrated Circuit (I2C), 1 RS485 serial communications; 2 serial interfaces, 21 Analogic/Digital (AD) inputs; 12 Digital Outputs, High Side Switch (2A/12 or 24 VDC) and 12 Digital Outputs Half-Bridge (2A/12 or 24 VDC).

On the other hand, we used also an M-Duino PLC Arduino $38 \mathrm{R}$ which provides: $8 \mathrm{AD}$ inputs, 4 interrupt inputs, 16 relays outputs $(220 \mathrm{~V} \mathrm{AC} / 5 \mathrm{~A}), 6 \mathrm{AD}$ outputs, 1 Ethernet interface, 1 USB port, 3 serial ports, 1 I2C, 1 RS485 port and 1 SPI port.

Controllino and M-Duino are similar controllers achieving the same functionalities, but M-Duino is used for industrial context, and Controllino is more used for a smart home context (Fig. 3). Libelium proposes specific shields in a wide range of applications domains which allows to implement easily several use cases and proof of concepts.

In terms of software, Node-Red [11] is used to provide a relationship between the different control chains. This application installed on the top of Node.Js [12] allows to inter-connect a system objects. Arduino IDE are used to program respectively Arduino and derived such as Controllino and M-Duino while Waspmote IDE is used to program Waspmote devices

\section{Methodology}

The learning protocol designed in our practical works is aiming to allow students to connect devices like Arduino and derived such as Waspmotes to a computer and to achieve some 

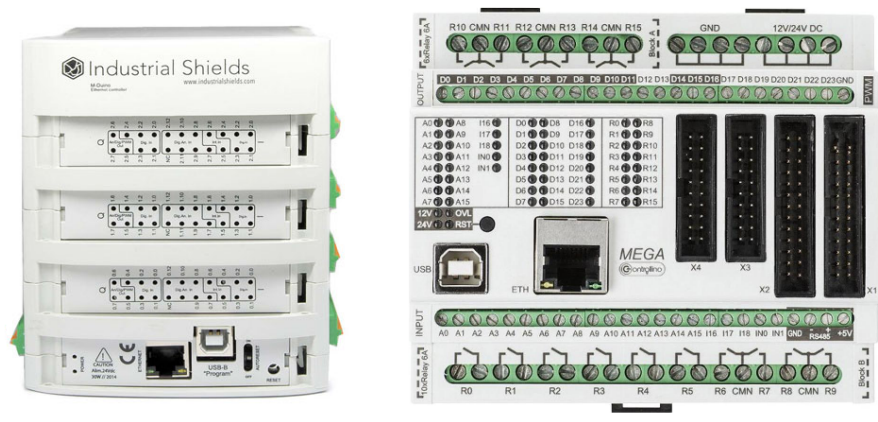

Fig. 3. M-Duino \& Controllino

lightinng actions according to the information gathered from the connected devices. In the remaining of this section we will describe the different steps of this process:

\section{A. Basic Arduino implementation}

The aim of this first experimentation is to familiarize students with the foundation of programming on Arduino and Arduino IDE manipulation. This stage needs a personal computer with Arduino IDE installed, 1 Arduino Uno, 1 breadboard, 1 monochromatic led, 1 resistor of $220 \mathrm{ohm}$, set of cables for breadboard male-male, 1 USB cable A male B male. At this stage, students use Arduino, which is directly connected to a personal computer by an USB cable. The first manipulation consists of implementing one monochromatic with a resistor in serial on an Arduino (Fig. 4) and then create a basic program to turn on the led. Then, students must blink the led by using of delay instruction.

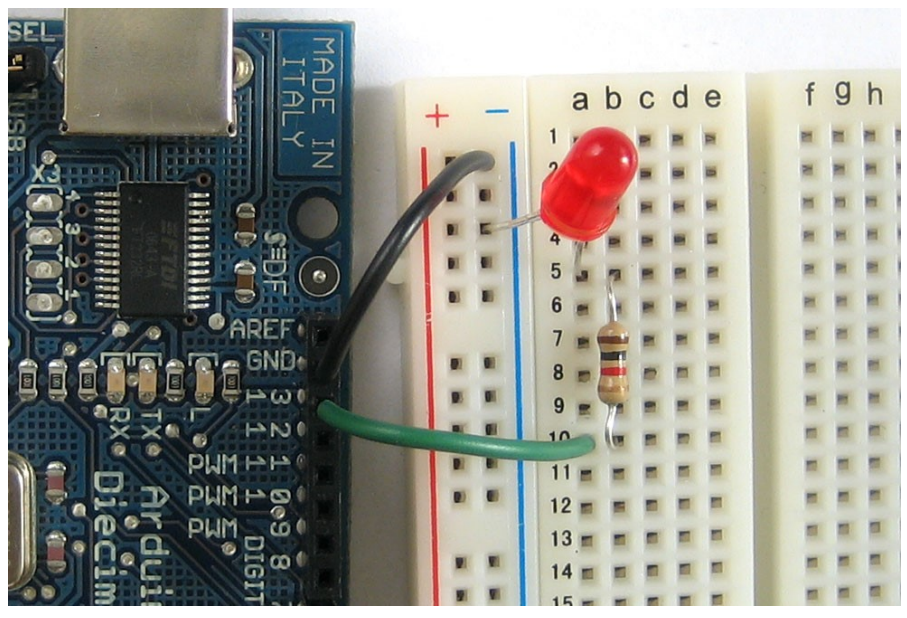

Fig. 4. Implantation of led and resistor on the breadboard

\section{B. Light control:}

Two experimentations are proposed to respectively control light in $\mathrm{DC}$ and $\mathrm{AC}$ tension. The first objective is to manipulate a multi-colored led in order to compose all the three fundamental colors and obtain a chosen color. The second aim is to manipulate a dimmer that allows to variate the intensity of the tension by using Pulse Width Modulation (PWM) output of
Arduino. Using PWM allows to explain the notion of analogic, digital and PWM (analogic emulate from digital output signal).

The material used for this second implementation is: 1 Arduino Uno, 1 shield RGB Velleman VMA01, 1 flat fine screwdriver and 1 dimmer $220 \mathrm{~V}$ AC with $112 \mathrm{w}$ incandescent bulb mount on its socket E14 (Fig. 2).

After inserting the RGB shield into the Arduino uno, it is necessary to implement the circuit illustrated at the Fig. 5. The Arduino is connected to the student personal computer and then a program is created to compose a chosen color by mixing the three primaries colors.

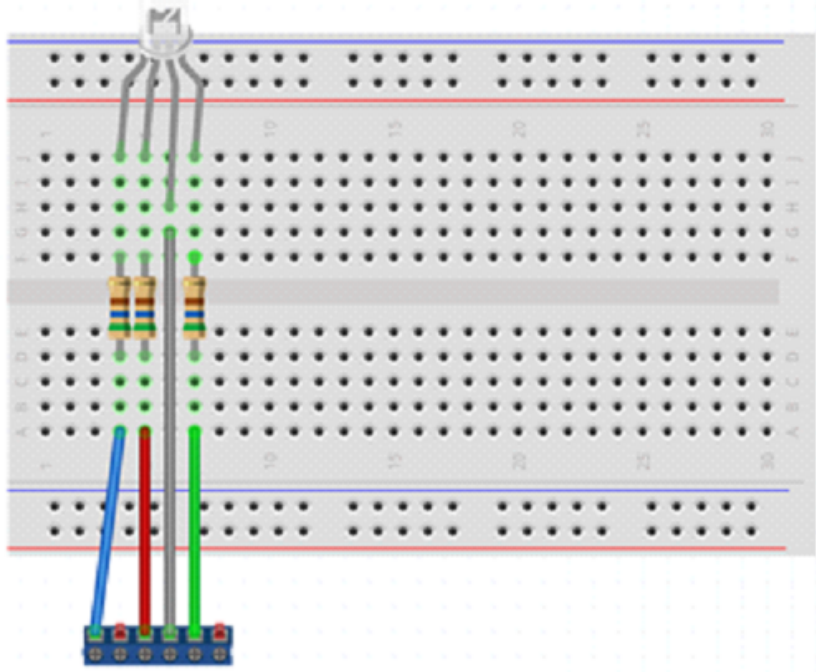

Fig. 5. Implantation of multicolor led, resistors and links with RGB shield

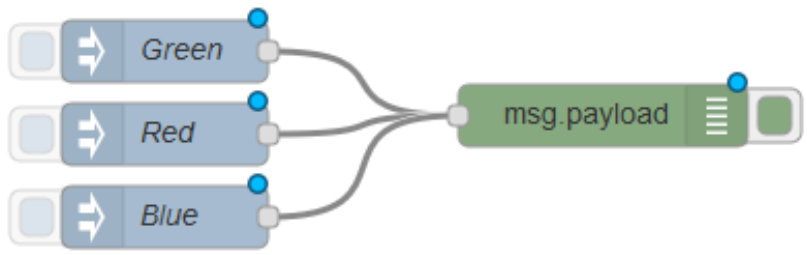

Fig. 6. Node-Red schema to send value of primary colors to RGB shield

The program is then modified to send the three colors from serial monitoring and in fact prepare the sending of instructions to the device. As shown in Fig. 6, Node-Red is used to send the value of each channel of RGB colors to the Arduino by serial connection.

The Arduino is disconnected with the PC and the shield is then extracted from the Arduino Uno. The dimmer 220v (Fig. 2 ) is then connected between the GND and a PWM pin. This particular output allows to generate continuous signals using on / off circuits; in the case of the Arduino, a digital output. The leaners can also connect the Arduino to the PC and create 
a program to variate the value of the analog signal and see the intensity of the bulb variate.

\section{Light control with M-duino and Controllino}

In the last steps, students have learned to manipulate an Arduino in particular analog, digital and PWM I/O. In the Internet of Things, actuators generally controlled remotely are used to perform actions on the external environment. Now, we will disconnect the microcontroller from the computer and interact with it by the Ethernet network to illustrate this concept.

The aim of this experimenation is to use Node-Red to control a led connected to an Ethernet device previously programmed to activate or deactivate a digital output upon reception of a determined character string.

In this stage, we will use: one M-Dunio, one Controllino, a set of LEDs $5 \mathrm{~mm} 50 \mathrm{mw}$, a set of resistors, a set of breadboards. Each group of students connect one led with it resistor place on a bread board between the GND and a digital output of the Ethernet device as shown on Fig. 7.

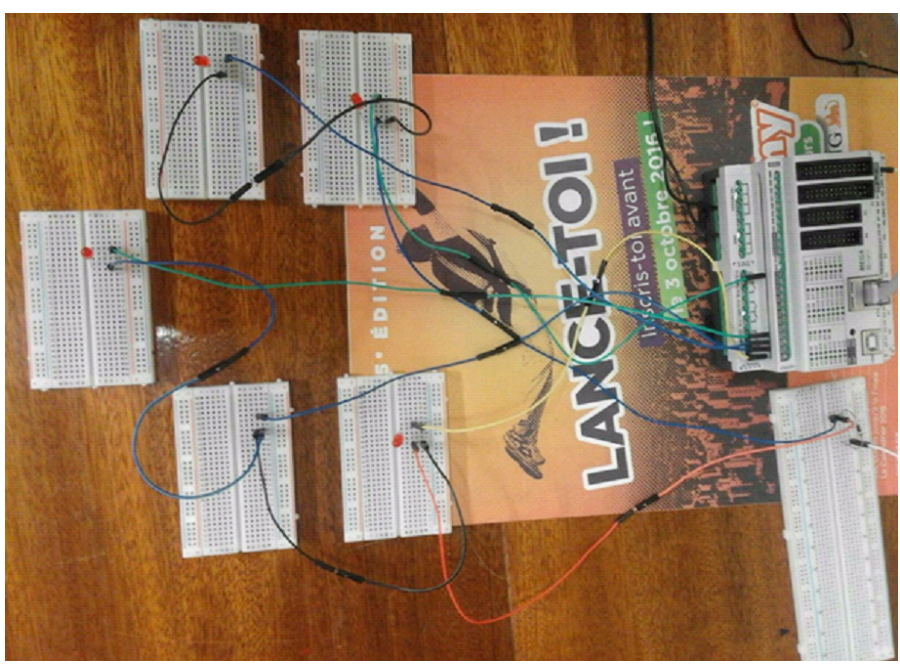

Fig. 7. Controllino with leds

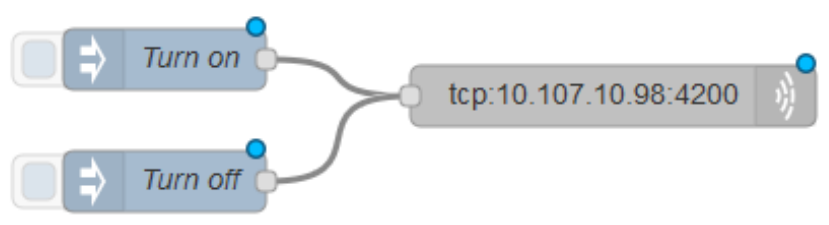

Fig. 8. Node-Red schema to turn on/off the Ethernet device

Each group of students received two strings of characters associate to the digital pin choosed to connect the led to the Ethernet device. These strings of characters must be send using Node-Red to the Ethernet device, one to turn on the led and the second to turn off it (Fig. 8).

\section{Connexion to external services}

In the previous section we have illustrated the notion of actuator. Theses actuators are activated in reaction to an event. To measure these events, we introduce the concept of sensors. The aim of this experimentation is to connect A Waspmote device with an external service (IFFTT) to send by Email the measured value of light intensity and the temperature of the class room.

This stage needs the following materials: a IFTTT ${ }^{1}$ account, one Waspmote version 1.5, one Light Dependent Resistor (LDR) or one ds $18 \mathrm{~b} 20^{2}$ temperature sensor, one USB cable micro A male A male. All this material is available in Evaluator Kit Libelium ${ }^{3}$ or Kit IoT Spartans Challenge Libelium ${ }^{4}$.

The (LDR or ds18b20) sensor is plugged with the Waspmote, which is then connected with the USB cable to computer. Students develop a little program to read LDR value or temperature value in the Waspmote IDE and upload it on the device. Then, they must launch Node-Red and compose a scheme to connect Waspmote in serial mode and send the payload to the IFTTT service, which send one Email contains temperature information (Fig. 9).

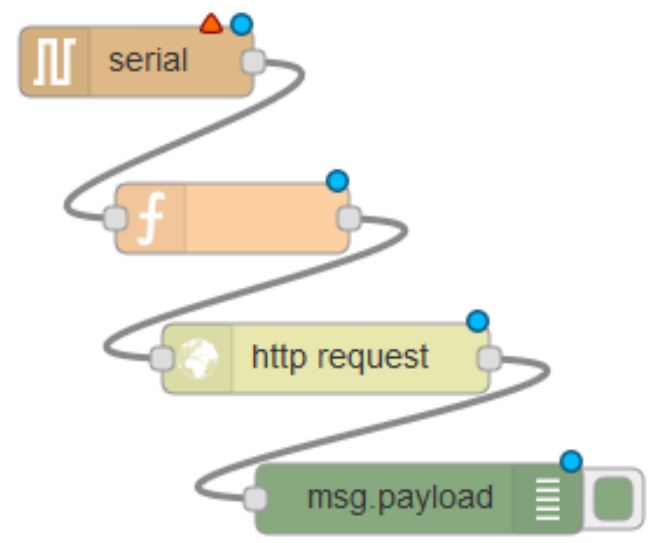

Fig. 9. Node-Red schema to connect serial data with IFTTT service

\section{E. Chain sensor to actuator}

In the both previous sections we have illustrated notions of sensors and actuators. Its now time to link them together and create a chain sensor-actuator.

The aim of this stage is to use Node-Red software in order to turn on/ off a $60 \mathrm{w}$ incandescent bulb when the intensity of the light measured by LDR implemented on the Waspmote decrease under a determinate threshold.

This part needs to use: 1 Waspmote, 1 Light Depend Resistor (LDR), Ethernet device: Controllino or M-Duino, 1 set of $60 \mathrm{w}$ incandescent bulb with socket, 1 USB cable A male micro A male.

\footnotetext{
${ }^{1}$ https://ifttt.com/discover

${ }^{2}$ https://datasheets.maximintegrated.com/en/ds/DS18B20.pdf

${ }^{3}$ https://www.cooking-hacks.com/waspmote-evaluator-kit

${ }^{4}$ https://www.cooking-hacks.com/waspmote-kit-iot-spartans-challenge
} 
The LDR sensor is plugged with the Waspmote which is then connected by USB cable to the computer. Leaners develop a program in Waspmote IDE to read LDR. Then they must launch Node-Red and compose a schema to connect Waspmote in serial mode. At the end of the chain, Node-Red send a character string by Ethernet protocol to the IP address of Controllino or M-Duino. Ethernet device are beforehand programmed to actuate or deactuate relay and turn on or turn off the incandescent bulb when it receives the correct character string.

\section{F. Using of $M Q T T$}

Internet of Things Network are low flow, it is therefore not possible to send many messages between sensors and all actuators. To limit the number of exchanged messages, the MQTT protocol is used. This service allows on one hand to have a value when the network is not available and to subscribe to a large range of actuators to one sensor. The material used in this stage is the same as section D with a Raspberry Pi Home Assistant. The aim of this experimentation is to introduce the use of a MQTT server.
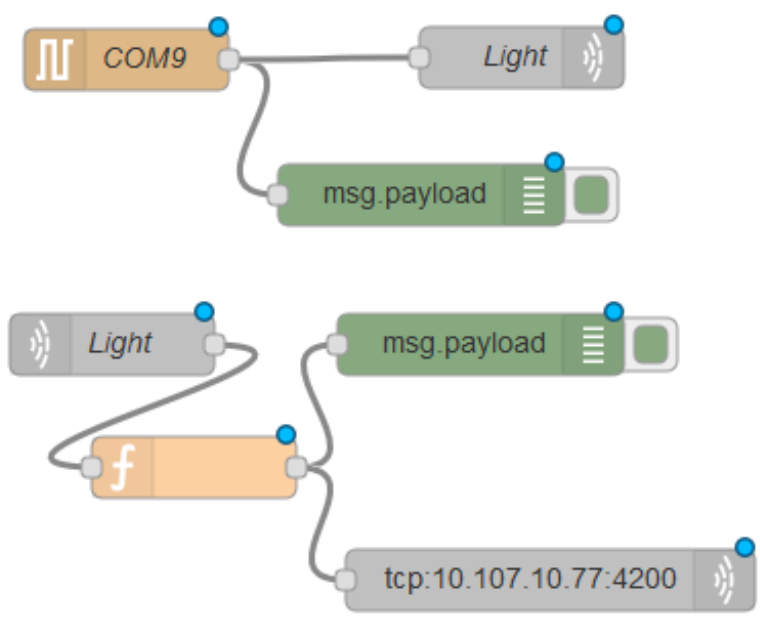

Fig. 10. Node-Red schema implementing MQTT intermediary

The MQTT server of Home Assistant is used to link to serial connection and the Ethernet device. As shown by Fig. 10, the schema illustrated at Fig. 10 is modified to add and intermediary MQTT server. Data coming from serial link with the Waspmote are publish on MQTT server. A subscription to MQTT server allows to retrieve data and verify if the threshold is reached and send a string chain to Ethernet device to turn on or turn off the relay.

\section{G. Connexion with Zigbee to a local server}

In the Internet of Things, objects generally communicate with wireless protocol. One of the more used protocol in SmartHome context is Zigbee.

The aim of this last experimentation is to use Wireless connection between Waspmote devices and a Gateway placed on a server. To realize this stage, we need: one Waspmote 1.5, one LDR and one Zigbee complementary module for Waspmote with its antenna. A USB Zigbee Gateway with another complementary module for Waspmote. The USB Zigbee gateway is connected to the server. The other module is plugged directly on the Waspmote which is connected to the computer with USB cable only the time to transfer the program. A software developed in $\mathrm{C} \#$ analyze data coming from Waspmote and update an xml file. a Node-Red schema is used to retrieve the $\mathrm{xml}$ output file provided by the gateway, to parse it and show data (Fig. 11).

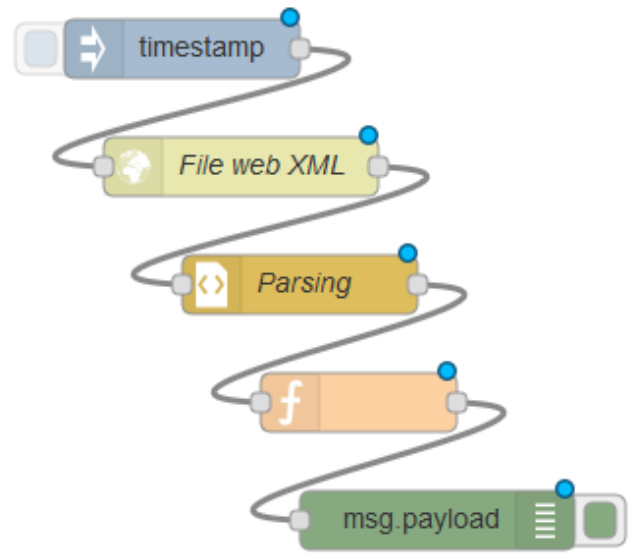

Fig. 11. Node-Red schema to parse data from xml file.

\section{H. Global presentation of Smart Home solution}

Finally, to illustrate the concept of Smart Home, we use the open source solution Home Assistant server hosted on a Raspberry Pi 3. A Pi Camera module is connected on the Raspberry Pi which host Home Assistant and allows an acquisition of images at rate of one by minute. As shown in Fig. 12, Arduino and Waspmote send sensors data to Home Assistant respectively by direct connection with an USB cable and by Zigbee transmission. Home assistant integrates also data from open data, external sources and Social network in a board accessible by a web browser on the local network. The board allows also to see the images taken by the Pi camera, to turn on / off actuators connected on the local network using M-Duino and Controllino.

\section{CONCLUSiON}

The learning protocol proposed in this paper lead students in a first time to be able to manipulate an Arduino directly connected to their own computer. This learning protocol use successively a monochromatic led and a multicolor led which allows from the mix of basic colors to produce a chosen color, and dimmer 220v to illustrate notions of analogic and digital signals. The notions of sensors, actuators and the link between sensors and actuators are illustrated. The connection between objects by means of wired and wireless is shown. Finally, external service, open data and MQTT protocol are 


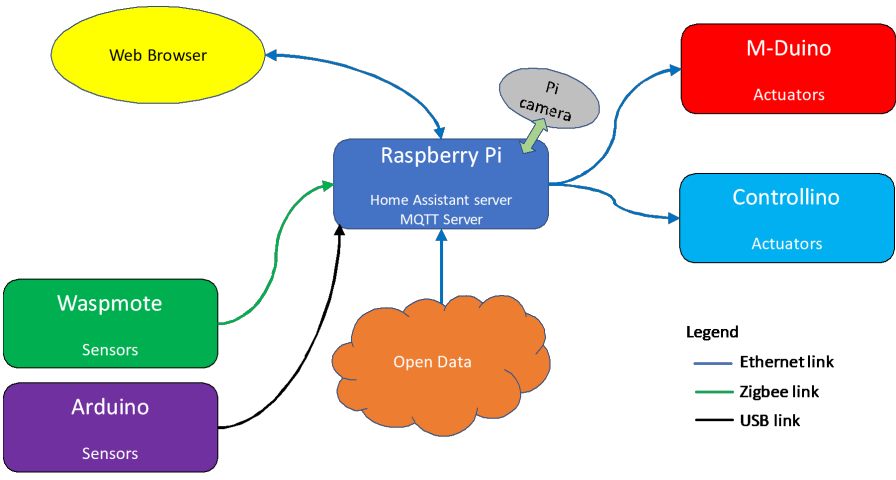

Fig. 12. General schema of your Smart Home system

integrated in an open source solution to give an integrate vision of a smart home system. The proposed protocol has been experienced with 1th Master Engineer students specialized in Architecture of the Faculty of Engineering at the University of Mons. Actually, a weakness of our approach is to be dependent of Waspmote Libelium with the risk of unavailability, disappearance or incompatibility with future versions of the hardware. The disappearance of Arduino or Libelium is less likely. Therefore, it is necessary to be independent of the manufacturers in order to perpetuate practical works.

As future work, we plan to exploit GPU Tegra Mobile Processors that offers high computation power thanks to the exploitation of GPU processors in parallel [13] [14]. In addition to the high performance, the parallel implementations would offer lower power consumption [15] as result of the fast treatment.

\section{ACKNOWLEDGMENT}

The authors would like to thank Mr Adriano Guttadauria for his technical support and for setting up all the electronic and informatic systems necessary for carrying out this learning protocol.

\section{REFERENCES}

[1] D. Evans. The internet of things: how the next evolution of the internet is changing everything. Technical report, White paper, CISCO, 2017.

[2] N. Shahid and S. Aneja. Internet of things: Vision, application areas and research challenges. In 2017 International Conference on I-SMAC (IoT in Social, Mobile, Analytics and Cloud) (I-SMAC), pages 583-587, 2017.

[3] B Billet. Systme de gestion de flux pour IInternet des objets intelligents. PhD Thesis, 2015. University of Versailles, Saint-Quentin-En-Yvelinnes (France), 2015.

[4] L. Atzori, A. Iera, and G. Morabito. From "smart objects" to "social objects": The next evolutionary step of the internet of things. IEEE Communications Magazine, 52(1):97-105, January 2014.

[5] S Cherrier. Architecture et protocole applicatifs pour la chorgraphie de services dans l'Internet des objets. PhD Thesis, 2013. University of Paris-East, France, 2013.
[6] J. Lin, W. Yu, N. Zhang, X. Yang, H. Zhang, and W. Zhao. A survey on internet of things: Architecture, enabling technologies, security and privacy, and applications. IEEE Internet of Things Journal, 4(5):1125-1142, Oct 2017.

[7] G. R. Gonzlez, M. M. Organero, and C. D. Kloos. Early infrastructure of an internet of things in spaces for learning. In 2008 Eighth IEEE International Conference on Advanced Learning Technologies, pages 381-383, July 2008.

[8] Jorge Gmez, Juan F. Huete, Oscar Hoyos, Luis Perez, and Daniela Grigori. Interaction system based on internet of things as support for education. Procedia Computer Science, 21:132 - 139, 2013.

[9] G. Carro Fernandez, E. Sancristobal Ruiz, M. Castro Gil, and F. Mur Perez. From rgb led laboratory to servomotor control with websockets and iot as educational tool. In Proceedings of 2015 12th International Conference on Remote Engineering and Virtual Instrumentation (REV), pages 32-36, Feb 2015.

[10] J. He, Dan Chia-Tien Lo, Y. Xie, and J. Lartigue. Integrating internet of things (iot) into stem undergraduate education: Case study of a modern technology infused courseware for embedded system course. In 2016 IEEE Frontiers in Education Conference (FIE), pages 1-9, Oct 2016.

[11] Michael Blackstock and Rodger Lea. Toward a distributed data flow platform for the web of things (distributed node-red). In Proceedings of the 5th International Workshop on Web of Things, WoT'14, pages 3439, New York, NY, USA, 2014. ACM.

[12] S. Tilkov and S. Vinoski. Node.js: Using javascript to build high-performance network programs. IEEE Internet Computing, 14(6):80-83, Nov 2010.

[13] S. A. Mahmoudi and P. Manneback. Multi-gpu based event detection and localization using high definition videos. In 2014 International Conference on Multimedia Computing and Systems (ICMCS), pages 81-86, April 2014.

[14] S. A. Mahmoudi and P. Manneback. Multi-cpu/multigpu based framework for multimedia processing. In Computer Science and Its Applications, pages 54-65, Cham, 2015. Springer International Publishing.

[15] S. A. Mahmoudi, M. A. Belarbi, S. Mahmoudi, and G. Belalem. Towards a smart selection of resources in the cloud for low-energy multimedia processing. Concurrency and Computation: Practice and Experience, 2017. 